field to enrich the sections on epidemiology, the prevention of drug misuse and the law and drug control policies. This is not the text to turn to for a detailed description of the neurobiology of drugs of misuse, or for an account of genetic theories of causation. Furthermore, despite its title, the book does not stray much beyond addiction to prescription and illegal drugs, although the inclusion of a chapter on alcohol is relevant in the current climate. However, when it comes to applying the evidence to the real-life clinical situations that face a psychiatrist working with people who use illegal drugs, this book reaches the parts that a systematic review-driven approach cannot always reach.

Ed Day Senior Clinical Lecturer in Addiction Psychiatry, Department of Psychiatry, University of Birmingham, Queen Elizabeth Psychiatric Hospital, Mindelsohn Way, Birmingham BI5 2QZ, UK

\section{Studies in the Assessment of Parenting}

Edited by Peter Reder, Sylvia Duncan \& Clare Lucey. Hove: Brunner-Routledge. 2003. 320 pp. $€ 17.95$ (pb).

ISBN I 583911804

Studies in the Assessment of Parenting aims to provide a practical guide to professionals who offer expert opinions to the courts in child care cases and to inform day-to-day work in child protection and in enhancing the care of children within their families. Most of the contributors are child and adolescent psychiatrists, but there are chapters by a psychologist, a forensic psychiatrist and a judge. The book is divided into four parts: 'Principles and practice', which offers a framework for assessment, including the problem of cultural matching between assessor and family; 'The child's perspective', in which attachment, significant dimensions of harm, the reliability of child witnesses and the views of children are addressed; 'Assessing parents', which focuses on specific parent groups, for example adolescents, violent individuals and parents with personality disorders; and 'Recommendations', which looks at parental denial, contact arrangements, foster care and adoption, and includes also a chapter in which an experienced judge outlines his role in weighing up a case and applying the law.

Much will be familiar territory for those working in child and adolescent mental health services (CAMHS). However, this book will be of value to higher trainees in CAMHS and other professionals new to this area of work. As a general adult psychiatrist increasingly being called upon to offer expert opinion in relation to maternal mental health in child care cases, I found much of it to be helpful and it will be invaluable to adult psychiatry colleagues who find themselves being asked for an expert opinion. However, other than a very good chapter by Christopher Cordess on parents with personality disorder, there is very little detail on parents with mental illness, which is often reduced to the phrase 'underlying psychopathology'. Concentrating on the legal system in England and Wales, the contributors only occasionally refer to equivalent legislation and almost never to alternative processes in other parts of the UK, which makes parts of this book less useful to professionals in those countries.

Carol Henshaw Senior Lecturer in Psychiatry, Keele University School of Medicine, Academic Suite, Harplands Hospital, Hilton Road, Stoke-on-Trent, Staffordshire ST4 6TH, UK

\section{Handbook of Affective Sciences}

Edited by Richard J. Davidson, Klaus R. Scherer \& H. Hill Goldsmith. Oxford: Oxford University Press. 2003. 1217 pp. $€ 130.00$ (hb). ISBN 0195126017

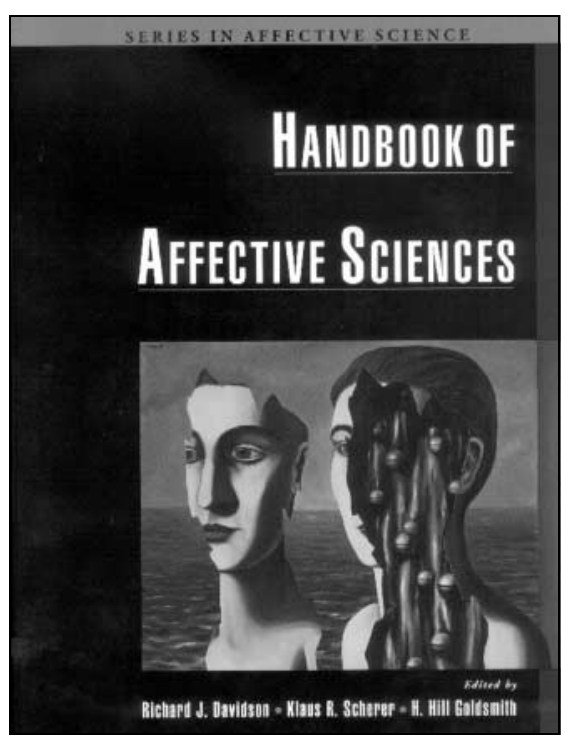

This is an outstanding textbook which summarises progress on research in the affective sciences up to about the year 2001. It is a remarkable piece of scholarship and the authors are to be congratulated on having the vision, determination and drive to carry through this project. The individual chapters are well written, but one of the great strengths of the book is that each section is introduced by an overview that draws together the main themes and each has been written with great clarity. There is also evidence of strong editorship in that the volume does not suffer from the usual problems of multiauthored texts with excessive overlap and repetition, and if there are contradictions these are highlighted rather than ignored.

The volume has an excellent introduction which sets the scene and explains the difficult task the authors had in deciding which areas to cover. The introduction contains working definitions of the various phenomena that were targets of enquiry within this handbook (emotions, mood, attitudes, affective style and temperament). The authors make the point that affective science is at the stage that cognitive psychology/neuroscience was at 20 years ago and which is now the most rapidly growing and largest multidisciplinary field in behavioural science today. One can imagine that the publication of this handbook may lead to a similar growth in affective science research.

The book starts with a review of the relevant neuroscience research, followed by accounts of the impact of genetics and development on affect. There are strong sections on the expression and cognitive components of emotion. Finally, the text deals with emotion and affect in their evolutionary, cultural and social contexts as well as their links with 'health' and with 'psychiatry'. Psychiatrists are often uncertain about what psychologists do and are particularly puzzled by the psychology questions in the MRCPsych examinations. Although this volume will not solve the former problem nor provide many answers for the latter, it reveals that conceptually and scientifically psychology research is well advanced and making a real impact on our understanding of complex and ramified topics.

Inevitably with such a large canvas there is some unevenness. I would have liked to have seen a lot more on the genetic underpinnings of emotion and affect but perhaps the editors have given this topic 
appropriate emphasis given the quality of the available evidence base. There has to be some delay in publication (perhaps not surprisingly, given the size and complexity of the volume) and the most recent references are to the year 2001. The field is expanding so rapidly that I urge the editors to start a new edition, constantly updating in a manner akin to painting the Forth Bridge.

Nicol Ferrier Professor of Psychiatry, University of Newcastle, School of Neurology, Neurobiology and Psychiatry, Royal Victoria Infirmary, Queen Victoria Road, Newcastle upon Tyne NEI 4LP, UK

\section{Substance Misuse in Psychosis. Approaches to Treatment and Service Delivery}

Edited by Hermine L. Graham, Alex Copello, Max J. Birchwood \& KimT. Mueser.

Chichester: Wiley. 2003. 420 pp. $€ 65.00$ (hb). ISBN 0 47I 492299

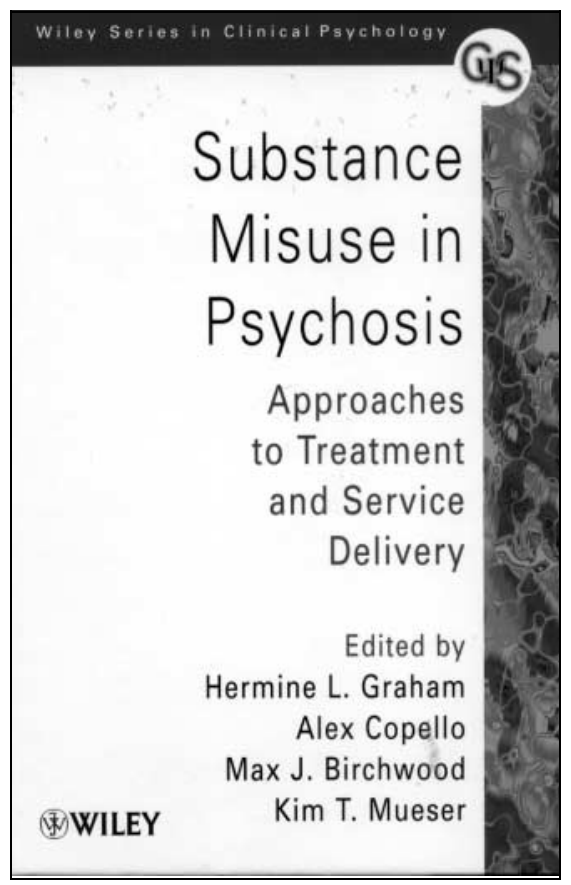

The high prevalence of alcohol and drug misuse in those with psychiatric illness is apparent to all who work within adult psychiatry. Traditionally, mental health and addiction services have operated in parallel. Differences between the two services in terms of organisation, geographical location and philosophy of care have often meant that patients have fallen between the two, leading to frustration for patients, families and staff. The past 10 years have seen increased understanding of the reasons for this comorbidity, the development of innovative integrated treatment services and the introduction of new treatments for substance misuse. This book provides a comprehensive review of these developments.

The book is divided into five parts. The first serves as an introduction, and begins with two chapters on the epidemiology of substance misuse in psychosis and the temporal relationship between the two. This is followed by three excellent chapters looking at family, social and cognitive conceptualisations, the first of which highlights the relationship between substance misuse in psychosis and the patient's family and social networks, including the role of expressed emotion. The sociological perspective that follows makes some interesting points in relation to stigma and the negative attitudes held not only by the public but also by clinicians managing this group of patients. In the first part's final chapter, Hermine L. Graham emphasises the importance of a cognitive formulation in managing patients, and how it can be used to generate hypotheses that can be tested and act as a guide for treatment interventions.

Part 2 reviews integrated service models, including community-based and inpatient treatment programmes. Examples are provided from the USA and the UK, including the Combined Psychosis and Substance Use (COMPASS) programme in north Birmingham. Part 3 considers assessment and treatment approaches such as cognitive-behavioural therapy, group therapy and family interventions. There is a single chapter on pharmacological approaches, which, although well written, is too short, with the result that many key areas are skimmed over. The editors should consider expanding this chapter in a future edition. Part 4 considers the needs of special populations, including the homeless and those with HIV/AIDS. Part 5 examines treatment outcome studies and looks towards future developments.

Over forty authors, mainly from the USA and the UK, have contributed. The editors have ensured that throughout the material remains clinically relevant, and many chapters employ illustrative case reports to good effect. This is a wellplanned and well-written book, and I recommend it.

Peter Haddad Consultant Psychiatrist, Cromwell House, Bolton, Salford and Trafford Mental Health Partnership, Eccles M30 OGT, UK

\section{Child Psychiatry and Psychology: An Introduction}

Edited by David H. Skuse. Abingdon: Medicine Publishing Company. 2003.

228 pp. $€ 29.00$ (pb). ISBN 0953259854

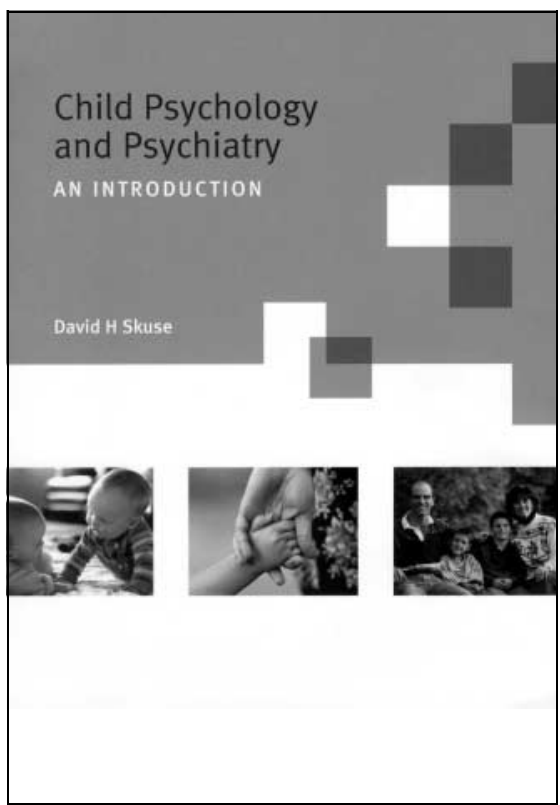

This book integrates developmental child psychology and an introduction to child psychiatry in a modestly sized guide to clinical practice by packing information into succinct chapters and excellent summarising tables. It spans normal development, assessment, family and genetic influences, classification, developmental and psychiatric disorders, management and treatment issues, and child psychiatry and the law. The authoritative contributors produce chapters that are easy to digest.

The assessment section offers excellent chapters on the assessment of psychiatric disorders in children, developmental paediatric and specialist neuropsychological assessment, IQ testing and scales to measure behavioural and emotional adjustment in children and their families. Summaries of other models that often contribute to a psychiatric assessment 